\title{
CRESCIMENTO INICIAL DO EUCALIPTO EM FUNÇÃO DA ACLIMATAÇÃO EM VIVEIRO
}

\author{
Jane Luísa Wadas Lopes ${ }^{1}$; João Carlos Cury Saad ${ }^{2}$; Iraê Amaral Guerrini ${ }^{3}$; Cristiano \\ Freitas Lopes ${ }^{4}$ \\ ${ }^{1}$ Eng $^{a}$ Florestal, Pós-doutoranda em Agronomia, Departamento de Engenharia Rural,UNESP/FCA. CP 237, \\ 18.603-970, Botucatu - SP, jane.luisa@uol.com.br; \\ ${ }^{2}$ Agrônomo, Livre Docente, Departamento de Engenharia Rural, UNESP/FCA - SP, joaosaad@fca.unesp.br; \\ ${ }^{3}$ Eng $^{0}$ Florestal, Professor Adjunto, Departamento de Recursos Naturais/Ciência do Solo, UNESP/FCA - SP, \\ iguerrini@fca.unesp.br; \\ ${ }^{4}$ Economista, Coordenador de Silvicultura, DURATEX - Unidade Florestal de Agudos, SP, \\ cristianoflopes@terra.com.br.
}

\section{RESUMO}

Este trabalho teve como objetivo avaliar os efeitos da aclimatação em viveiro, das mudas de Eucalyptus grandis vs. Eucalyptus urophylla, no crescimento inicial em dois solos de SP, um arenoso e outro argiloso. As mudas foram produzidas nos substratos Plantmax e casca de arroz carbonizada mais vermiculita, e manejadas, a partir dos 60 dias após estaquia (DAE), durante a fase de rustificação, com cinco diferentes frequências de irrigação por subsuperfície, restabelecendo a condição de capacidade de campo: F1, F2, F3 e F4, as quais foram irrigadas uma, duas, três e quatro vezes ao dia, respectivamente, e FD, mantidas em irrigação, até o plantio aos 90 DAE. Num delineamento de campo de blocos ao acaso com quatro repetições, foram realizadas avaliações da altura da parte aérea (HPA), aos 6 e aos 13 meses após o plantio e do diâmetro à altura do peito (DAP) aos 13 meses após o plantio. Verificou-se que não houve influência no crescimento, tanto em altura como em diâmetro, para ambos os solos, do manejo da água na fase de rustificação das mudas.

Palavras Chave: implantação florestal, irrigação por subsuperfície, manejo da água.

\section{LOPES, J.L.W.; SAAD, J.C.C.; GUERRINI, I.A.; LOPES, C.F. EUCALYPT INITIAL GROWTH IN RESPONSE TO NURSERY ACCLIMATIZATION}

\section{ABSTRACT}

This study aimed to evaluate the acclimatization effects in the Eucalyptus grandis vs. Eucalyptus urophylla seedlings nursery in their initial growth in two soils types, clay and sandy. The seedlings were planted in Plantmax substrate and in rice hulls plus vermiculite, and managed, after 60 days of the mass propagation (DAE), during the rustication. There were five different frequencies of subsurface drip irrigation, restoring the soil field capacity condition: F1, F2, F3 and F4, which were irrigated once, twice, three and four times a day, respectively, and FD, kept in continue irrigation until planting at $90 \mathrm{DAE}$. In a randomized block design with four replications, plant height (HPA) were evaluated at 6 and 13 months after planting and the diameter at breast height (DAP) at 13 months after planting. Findings show that water management at hardening phase seedlings had no influence on growth in both soils. 
KEYWORDS: deployment forest, underwater irrigation, water management

\section{INTRODUÇÃO}

Atualmente o Brasil possui tecnologia de implantação, condução e colheita de plantações de eucalipto (Couto et al., 2002), que estão entre os ecossistemas mais produtivos do mundo (Silva \& Matos, 2003). No entanto, a rusticidade de mudas continua sendo um assunto controverso (Neves, 2004). Discute-se sua importância para a garantia da sobrevivência dos plantios e no arranque da floresta, em condições climáticas adversas (Carneiro, 1995; Stape et al., 2001), bem como, o próprio entendimento do que seja rustificação. Também se discute o fato de que mudas aclimatadas de modo distinto, desenvolvam-se com diferenças (Stape et al., 2001), uma vez que o crescimento do eucalipto parece estar muito mais relacionado aos atributos químicos do solo, ao preparo do terreno, à escolha das espécies, ao grau de melhoramento genético, ao manejo e aos tratos culturais. $\mathrm{O}$ cultivo mínimo, por sua vez poderia contribuir para uma maior heterogeneidade de crescimento (Gonçalves et al. 1996; Gava, 2002), principalmente em se tratando de solos com texturas diferentes, o que por sua vez, traria implicações econômicas ao investimento, com produtividades abaixo do esperado.

Várias pesquisas trataram sobre relações hídricas em eucalipto, buscando dentre outros fatores, a rusticidade de mudas na sobrevivência em campo (Sasse et al., 1996; Silva et al. 2003; Lopes et al., 2005; Pereira et al., 2006). Poucas, no entanto, abordam as fases de plantio e de como ocorre o crescimento em função da muda ser mais ou menos aclimatada às adversidades de campo, principalmente no que diz respeito à umidade do solo, o que faz com que muitas não sobrevivam, implicando em replantios e em povoamentos heterogêneos. Desse modo, estudos sobre aclimatação de mudas de eucalipto através do manejo da água em viveiro, podem fornecer ferramenta importante para o desenvolvimento inicial da floresta.

O objetivo desse trabalho foi verificar os efeitos da aclimatação, realizada durante a fase de rustificação das mudas, do clone $\mathrm{H} 13$ de Eucalyptus grandis vs. Eucalyptus urophylla, no desenvolvimento inicial da floresta no primeiro ano de vida em dois tipos de solo, arenoso e argiloso.

\section{MATERIAL E MÉTODOS}

O experimento foi conduzido de novembro de 2005 a fevereiro de 2007, no estado de SP. A aclimatação das mudas através dos manejos hídricos foi conduzida em Patrocínio Paulista, onde também ocorreu o plantio em solo arenoso, e em Guará, em solo argiloso.

As mudas do clone H13 de Eucalyptus grandis vs. Eucalyptus urophylla, um dos clones impulsionadores do ritmo de desenvolvimento florestal brasileiro, alcançado segundo Carvalho (2000), pelo bom crescimento, característica do Eucalyptus grandis e pela rusticidade, característica do Eucalyptus urophylla, foram produzidas em recipientes plásticos de uso tradicional na produção de mudas florestais, do tipo tubetes, com capacidade para 50 $\mathrm{cm}^{3}$, através da técnica de miniestaquia. A estaquia se deu em dois substratos, sendo a produção de 5000 mudas com Plantmax estacas (PLX) e, 5000 mudas com a mistura em partes iguais de casca de arroz carbonizada e vermiculita (CACV).

Aos 60 DAE foram acondicionadas mudas de mesmos padrões de qualidade (altura e diâmetro de colo) e idade, em bandejas do tipo caixa, na densidade de 200 plantas $\mathrm{m}^{-2}$. Dessa 
forma, foi constituído um delineamento inteiramente casualizado com 5 tratamentos de 1000 mudas para cada substrato, com 4 repetições de 250 plantas cada, irrigados por subsuperfície (restabelecendo a condição de capacidade de campo dos substratos), na fase de rustificação das mudas, conforme é apresentado na Tabela 1 e na Figura 1.

Tabela 1. Tratamentos hídricos aplicados diariamente às mudas, durante a fase de rustificação.

\begin{tabular}{|c|c|c|c|}
\hline Tratamento & Substrato & $\begin{array}{l}\text { Frequência da } \\
\text { irrigação (vezes } \text { dia }^{-1} \text { ) }\end{array}$ & Horário da irrigação \\
\hline F1 & $\begin{array}{l}\text { CACV e } \\
\text { PLX }\end{array}$ & uma vez & $13 \mathrm{~h} 00$ \\
\hline F2 & $\begin{array}{l}\text { CACV e } \\
\text { PLX }\end{array}$ & duas vezes & $10 \mathrm{~h} 30$ e $16 \mathrm{~h} 30$ \\
\hline $\mathrm{F} 3$ & $\begin{array}{l}\text { CACV e } \\
\text { PLX }\end{array}$ & três vezes & $09 \mathrm{~h} 30,13 \mathrm{~h} 30$ e $17 \mathrm{~h} 30$ \\
\hline $\mathrm{F} 4$ & $\begin{array}{l}\text { CACV e } \\
\text { PLX }\end{array}$ & quatro vezes & $8 \mathrm{~h} 00,11 \mathrm{~h} 00,14 \mathrm{~h} 00$ e $17 \mathrm{~h} 00$ \\
\hline FD & $\begin{array}{l}\text { CACV e } \\
\text { PLX }\end{array}$ & \multicolumn{2}{|c|}{$\begin{array}{c}\text { mantidas em lâmina de água de } 2 \mathrm{~cm} \text { de altura na base do } \\
\text { tubete }\end{array}$} \\
\hline
\end{tabular}

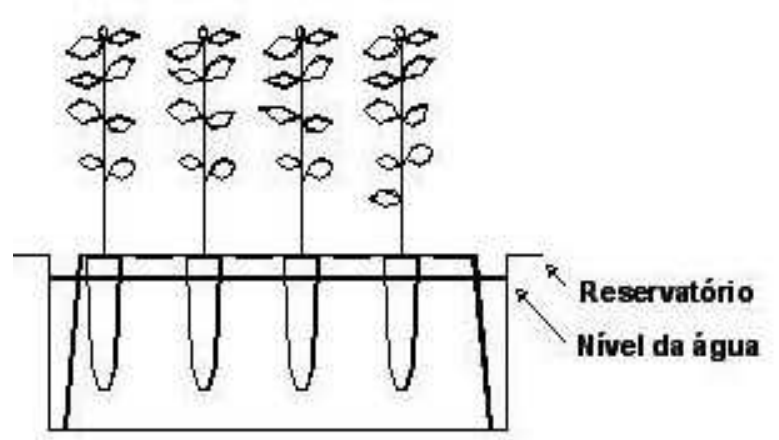

a

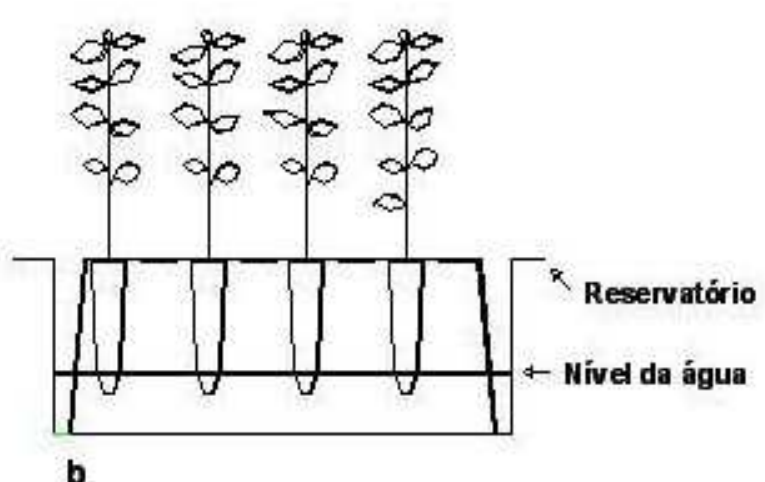

Figura 1. Representação do sistema de irrigação para os manejos F1, F2, F3 e F4 (a) e para o manejo FD (b).

No tratamento FD, os substratos eram continuamente mantidos na condição de capacidade de campo pela conservação do nível de água $2 \mathrm{~cm}$ acima da borda inferior do tubete. Nos tratamentos F1 a F4, cada irrigação correspondia ao restabelecimento da condição de umidade equivalente à capacidade de campo, o que era obtido elevando-se nível de água até $2 \mathrm{~cm}$ abaixo da borda superior do tubete, mantendo-se neste nível por no mínimo 15 minutos, de tal forma a assegurar que a frente de molhamento atingisse a superfície superior do substrato.

Durante a fase de rustificação as mudas em ambos os substratos foram adubadas duas vezes por semana, com $50 \mathrm{~g}$ de cloreto de cálcio, 33,3 g de fosfato monoamônio, $140 \mathrm{~g}$ de cloreto de cálcio e 28,0 g de sulfato de amônia, dissolvidos em água, formando uma calda de 
70 litros, suficientes para 7000 mudas.

A lâmina média diária bruta de água em função dos substratos e dos manejos hídricos durante a fase de rustificação das mudas foi calculada para as frequências F1, F2, F3 e F4 através de pesagens e para a frequência FD, a lâmina foi obtida através da leitura em escala graduada. As características morfológicas, altura de parte aérea (HPA), diâmetro de colo (DC), relação entre altura de parte aérea e diâmetro de colo (Rel H:D), área foliar (AF), número de ramos (NR) e pares de folhas (NPF), em função dos substratos e do manejo hídricos ao final da rustificação foram determinadas através de régua numerada, paquímetro digital Starret -modelo 727-2001 e medidor de AF LI-COR -modelo LI-3000 A, usado para definir a $\mathrm{AF}$ e para o ajuste da equação de regressão linear usada no cálculo das $\mathrm{AF}$, posterior à digitalização das imagens das folhas, e papel milimetrado para comparação desta metodologia.

Após os 90 DAE, 4.000 mudas de cada substrato foram selecionadas para o plantio em um espaçamento de $3 \mathrm{~m}$ entre linhas e $2 \mathrm{~m}$ entre plantas $(3 \times 2)$, totalizando 1.667 plantas ha ${ }^{-1}$, em dois solos texturalmente distintos, arenoso com 847, 117 e $36 \mathrm{~g} \mathrm{~kg}^{-1}$ de areia, silte e argila respectivamente, em Patrocínio Paulista, onde anteriormente ao eucalipto era realizada a pecuária extensiva de leite, e outro, argiloso com 70, 331 e $599 \mathrm{~g} \mathrm{~kg}^{-1}$ de areia, silte e argila respectivamente, em Guará, onde anteriormente ao eucalipto era praticado o cultivo intensivo de soja. Em ambos os locais de plantio, foram realizadas as operações de preparo do terreno, como o rebaixamento da vegetação (usando trator de pneu com roçadeira, somente no solo de textura argilosa), o controle da matocompetição (através da aplicação de herbicida em área total), o combate à formigas (gêneros Acromyrmex e Atta) e cupins, e uma subsolagem na linha de plantio à $50 \mathrm{~cm}$ de profundidade usando trator de pneu e subsolador de arrasto.

Antes das mudas serem plantadas tiveram seus sistemas radiculares imersos em uma solução à $1 \%$ de fosfato monoamônio e à $0,25 \%$ de cupinicida à base de fipronil. $\mathrm{O}$ plantio foi manual usando plantadeira tipo matraca, sendo a primeira irrigação efetuada logo em seguida, através de trator de pneu e carreta pipa, colocando-se $2 \mathrm{~L}$ de água por cova de plantio. Após 4 dias efetuou-se uma segunda irrigação com a mesma quantia de água. A umidade gravimétrica do solo, no momento de plantio era de $10,5 \%$ (camada de $0-20 \mathrm{~cm}$ ) e de $11,9 \%$ (camada de $20-40 \mathrm{~cm}$ ) para o arenoso, e de $23,1 \%$ (camada de $0-20 \mathrm{~cm}$ ) e de $26,8 \%$ (camada de $20-40 \mathrm{~cm}$ ) para o argiloso.

Com base na análise de fertilidade realizada nos dois solos (Tabela 2), em ambos os locais de plantio foi realizada a correção nutricional, sendo que a dosagem para cada local, de $40 \mathrm{Kg}$ de $\mathrm{N} \mathrm{ha}^{-1}$ para o argiloso e 60,40 e $50 \mathrm{Kg} \mathrm{ha}^{-1}$ de N-P-K, respectivamente para o arenoso, foi determinada segundo as recomendações de Gonçalves et al. (1996), conforme detalhamento apresentado na Tabela 3. A calagem não foi realizada, devido às dúvidas que existem em relação às respostas do $\mathrm{Ca}$ em eucalipto (Barros et al., 1990).

O delineamento estatístico adotado foi o de blocos ao acaso com 4 repetições, sendo que em cada parcela foram plantadas 96 mudas dispostas em 8 linhas, com 12 plantas em cada uma delas, obedecendo a mesma distribuição espacial para os tratamentos em ambos os locais, com parcelas úteis representadas pelas 24 plantas centrais. Desse modo, em ambos os solos foram plantadas as mudas de todos os tratamentos conduzidos na fase de viveiro, obedecendo à um mesmo arranjo espacial.

Aos 6 e aos 13 meses após o plantio foram realizadas as mensurações nas parcelas úteis em cada um dos locais de plantio. Aos 6 meses foram mensuradas as alturas das árvores, com auxílio de vara graduada e aos 13 meses, além da altura, foram também mensurados os diâmetros à altura do peito (DAP a 1,30 m), com auxílio de paquímetro digital. 
Tabela 2. Resultados das análises de fertilidade dos solos.

\begin{tabular}{|c|c|c|}
\hline \multirow{2}{*}{ Determinações } & \multicolumn{2}{|c|}{ Tipos de solo } \\
\hline & Textura arenosa & Textura argilosa \\
\hline $\mathrm{pH}\left(\mathrm{CaCl}_{2}\right)$ & 4,4 & 4,9 \\
\hline Matéria Orgânica $\left(\mathrm{g} \mathrm{dm}^{-3}\right)$ & 12,0 & 28,0 \\
\hline$P$ resina $\left(\mathrm{mg} \mathrm{dm}^{-3}\right)$ & 4,0 & 132,0 \\
\hline $\mathrm{Al}^{3+}\left(\mathrm{mmol}_{\mathrm{c}} \mathrm{dm}^{-3}\right)$ & 5,0 & 2,0 \\
\hline $\mathrm{H}+\mathrm{Al}^{+}\left(\mathrm{mmol}_{\mathrm{c}} \mathrm{dm}^{-3}\right)$ & 35,0 & 46,0 \\
\hline $\mathrm{K}^{+}\left(\mathrm{mmol}_{\mathrm{c}} \mathrm{dm}^{-3}\right)$ & 0,4 & 2,3 \\
\hline $\mathrm{Ca}^{+}\left(\mathrm{mmol}_{\mathrm{c}} \mathrm{dm}^{-3}\right)$ & 8,0 & 32,0 \\
\hline $\mathrm{Mg}^{+}\left(\mathrm{mmol}_{\mathrm{c}} \mathrm{dm}^{-3}\right)$ & 2,0 & 7,0 \\
\hline $\mathrm{SB}^{+}\left(\mathrm{mmol}_{\mathrm{c}} \mathrm{dm}^{-3}\right)$ & 11,0 & 42,0 \\
\hline $\mathrm{CTC}^{+}\left(\mathrm{mmol}_{\mathrm{c}} \mathrm{dm}^{-3}\right)$ & 46,0 & 87,0 \\
\hline $\mathrm{V} \%{ }^{+}\left(\mathrm{mmol}_{\mathrm{c}} \mathrm{dm}^{-3}\right)$ & 24,0 & 48,0 \\
\hline $\mathrm{S}\left(\mathrm{mg} \mathrm{dm}^{-3}\right)$ & 4,0 & 10,0 \\
\hline $\mathrm{B}\left(\mathrm{mg} \mathrm{dm}^{-3}\right)$ & 0,21 & 0,55 \\
\hline $\mathrm{Cu}\left(\mathrm{mg} \mathrm{dm}^{-3}\right)$ & 1,2 & 4,3 \\
\hline $\mathrm{Fe}\left(\mathrm{mg} \mathrm{dm}^{-3}\right)$ & 38,0 & 34,0 \\
\hline $\operatorname{Mn}\left(\mathrm{mg} \mathrm{dm}^{-3}\right)$ & 5,0 & 4,7 \\
\hline $\mathrm{Zn}\left(\mathrm{mg} \mathrm{dm}^{-3}\right)$ & 0,2 & 4,6 \\
\hline
\end{tabular}

Tabela 3. Adubação realizada nas áreas de plantio.

\begin{tabular}{|c|c|c|c|c|c|}
\hline \multirow{3}{*}{ Local } & \multicolumn{5}{|c|}{ Fonte de nutrientes } \\
\hline & $\begin{array}{c}6: 30: 6+0,5 \% \\
\mathrm{Zn}^{1}\end{array}$ & \multicolumn{2}{|c|}{$\begin{array}{c}13: 00: 15+0,5 \% \mathrm{~B}^{1} \\
\left(\mathrm{~kg} \mathrm{ha}^{-1}\right)\end{array}$} & \multicolumn{2}{|c|}{$\mathrm{NH}_{4} \mathrm{SO}_{4}{ }^{1}$} \\
\hline & Plantio & $1^{\mathrm{a}}$ cobertura & $2^{a}$ cobertura & Plantio & $1^{\mathrm{a}}$ cobertura \\
\hline $\begin{array}{l}\text { Patrocínio } \\
\text { Paulista }\end{array}$ & 200 & 200 & 200 & 0 & 0 \\
\hline Guará & 0 & 0 & 0 & 100 & 100 \\
\hline
\end{tabular}

(1) aplicado localizadamente.

Durante o período de avaliação do experimento foram considerados os dados de precipitação mensais fornecidos pela CAROL - Cooperativa dos Produtores da Região de 
Orlândia, SP, das estações mais próximas aos plantios, de $1 \mathrm{~km}$ para o solo argiloso e de 10 $\mathrm{km}$ para o solo arenoso.

Para fins de comparação entre os resultados das variáveis mensuradas, foi usada a análise de variância, sendo as médias comparadas pelo Teste de Tukey ao nível de $5 \%$ de probabilidade.

\section{RESULTADOS E DISCUSSÃO}

Durante a fase de viveiro, as mudas produzidas no substrato CACV consumiram as maiores lâminas médias diárias brutas de água, exceto nas condições consideradas como de falta de água, caso das F1 e de disponibilidade de água, caso das FD, onde o consumo bruto foi igual estatisticamente (Tabela 4). Possivelmente o consumo maior em CACV se deva às suas características físicas, de menor retenção de água em relação ao PLX, uma vez que sua formulação é de $50 \%$ de casca de arroz carbonizada e 50\% de vermiculita, enquanto que a formulação do PLX é de $40 \%$ de casca de pinus compostada, 20\% de vermiculita fina, 20\% de vermiculita super fina e $20 \%$ de turfa.

Tabela 4. Lâmina média diária bruta de água, em função dos substratos e dos manejos hídricos durante a fase de rustificação das mudas.

\begin{tabular}{ccccc}
\hline \multirow{2}{*}{$\begin{array}{c}\text { Manejo } \\
\text { hídrico }\end{array}$} & \multicolumn{4}{c}{ Lâmina média bruta de água $\left(\mathrm{mm} \mathrm{dia}^{-1}\right)$} \\
\cline { 2 - 5 } & Substrato CACV & Substrato PLX & D.M.S. & C.V. \\
\hline F1 & $3,50 \mathrm{Ae}$ & $3,90 \mathrm{Ad}$ & 0,38 & 2,92 \\
F2 & $5,50 \mathrm{Ad}$ & $5,00 \mathrm{Bc}$ & 0,52 & 2,79 \\
F3 & $7,40 \mathrm{Ab}$ & $6,30 \mathrm{Bb}$ & 1,78 & 0,43 \\
F4 & $8,50 \mathrm{Aa}$ & $7,40 \mathrm{Ba}$ & 3,37 & 0,94 \\
FD & $6,30 \mathrm{Ac}$ & $5,40 \mathrm{Abc}$ & 1,50 & 7,28 \\
\hline D.M.S. & 0,70 & 1,07 & & \\
\hline C.V. & 3,83 & 3,26 & & \\
\hline
\end{tabular}

D.M.S.: diferença mínima estatística do Teste de Tukey $(\alpha=5 \%)$; C.V.: coeficiente de variação. Médias seguidas de letras minúsculas iguais na mesma coluna e maiúsculas na mesma linha, não diferem pelo Teste de Tukey, ao nível de 5\% de significância.

Apesar de na fase de viveiro terem ocorrido 8\% de mortes das mudas F1 de CACV, os padrões de qualidade das plantas, representados apenas pelas suas características morfológicas (Tabela 5), foram considerados como acima do limite mínimo estabelecido para o plantio de mudas de eucalipto (Gomes et al., 1996).

Embora a umidade inicial tenha sido maior no solo argiloso, os índices de chuva (Figura 2) de janeiro a março de 2006, foram maiores no arenoso, com média mensal de 343,6 $\mathrm{mm}$ de chuva, em detrimento do argiloso, de $262,7 \mathrm{~mm}$ de chuva, o que, por sua vez, indica que em ambos os locais o volume foi acima do requerido. De abril a outubro de 2006, os índices se assemelharam nos dois locais de plantio, e de outubro de 2006 até janeiro de 2007 os índices foram maiores no solo argiloso, resultando em $141,6 \mathrm{~L} \mathrm{~m}^{-2}$ a mais de água neste solo para o 
período. Embora tenham ocorrido estas diferenças pode-se afirmar que não houve falta de água para a plantação de um local em relação a do outro, representando de janeiro a julho de 2006, lâmina média diária de 4,2 $\mathrm{L} \mathrm{m}^{-2}$ na área argilosa e $5,4 \mathrm{~L} \mathrm{~m}^{-2}$ na arenosa, e de agosto a dezembro de 2006, lâmina média diária de $9,2 \mathrm{~L} \mathrm{~m}^{-2}$ para a área argilosa e $6,5 \mathrm{~L} \mathrm{~m}^{-2}$ para a arenosa.

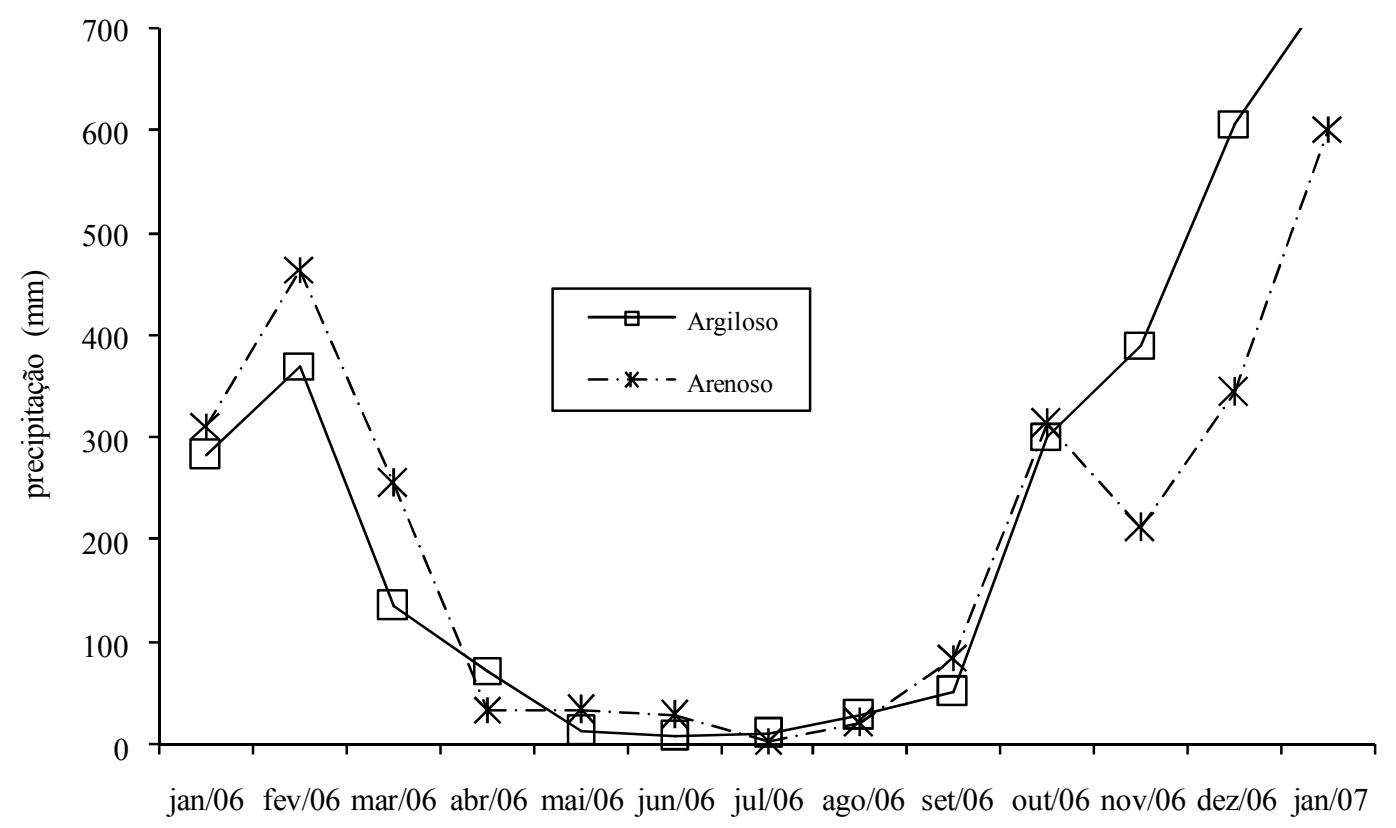

período

Figura 2. Representação gráfica da precipitação mensal (mm) ocorrida nos dois locais de plantio, de janeiro de 2006 a janeiro de 2007.

Em ambos os solos, tanto aos seis, como aos 13 meses de idade, o desenvolvimento em altura da plantação de eucalipto não foi influenciado pelos substratos e pela aclimatação das mudas através dos manejos hídricos (Tabelas 6, 7,8 e 9), realizada durante a fase de rustificação no viveiro.

Mudas aclimatadas à condição de déficit hídrico, como podem ser consideradas as F1 em ambos os substratos, poderiam ter apresentado diferenças de crescimento em campo, principalmente até os seis meses de idade, quando comparadas àquelas que não foram aclimatadas, caso principalmente das $\mathrm{F} 4$, onde o consumo bruto de água, durante a rustificação, foi significativamente superior quando comparado ao consumo das F1 (Tabela 2). Tais constatações confirmam que a qualidade das mudas tem efeito significativo apenas na sobrevivência inicial dos plantios (Carneiro, 1995), e que esta por sua vez, tem expressivas implicações na heterogeneidade do povoamento com conseqüências na sua produtividade, justamente devido aos replantios, muitas vezes realizados tardiamente. Desta forma, neste experimento, o fato das mudas terem sido produzidas em substratos distintos e de terem sido submetidas, durante a rustificação, a diferentes condições hídricas, não promoveu prejuízos à plantação, representados pelo crescimento em altura (Tabelas 6, 7,8 e 9) e em diâmetro (Tabelas 8 e 9) nos dois locais de plantio. 
Tabela 5. Resultados médios de altura de parte aérea (HPA), diâmetro de colo (DC), relação entre altura de parte aérea e diâmetro de colo (Rel H:D), área foliar (AF), número de ramos (NR) e pares de folhas (NPF) para as mudas de Eucalyptus grandis vs. Eucalyptus urophylla, em função dos substratos e do manejo hídricos ao final da rustificação.

\begin{tabular}{|c|c|c|c|c|c|c|c|c|}
\hline \multirow{3}{*}{$\begin{array}{l}\text { Manejo } \\
\text { hídrico }\end{array}$} & \multicolumn{4}{|c|}{ HPA (cm) } & \multicolumn{4}{|c|}{$\mathrm{DC}(\mathrm{mm})$} \\
\hline & \multicolumn{2}{|c|}{ Substrato } & \multirow{2}{*}{$\begin{array}{c}\text { D.M.S } \\
\text {. }\end{array}$} & \multirow[b]{2}{*}{ C.V. } & \multicolumn{2}{|c|}{ Substrato } & \multirow{2}{*}{$\begin{array}{c}\text { D.M. } \\
\text { S. }\end{array}$} & \multirow[b]{2}{*}{ C.V. } \\
\hline & $\mathrm{CAC}$ & PLX & & & $\mathrm{CAC}$ & PLX & & \\
\hline \multirow[t]{2}{*}{ F1 } & 25,78 & 24,75 & 3,16 & 5,57 & $2,98 \mathrm{Ab}$ & $2,86 \mathrm{Aa}$ & 0,52 & 7,86 \\
\hline & $\mathrm{Ab}$ & $\mathrm{Ab}$ & & & & & & \\
\hline \multirow[t]{2}{*}{$\mathrm{F} 2$} & 31,10 & 24,52 & 1,31 & 2,10 & $3,42 \mathrm{Aa}$ & $2,90 \mathrm{Ba}$ & 0,27 & 3,62 \\
\hline & $\mathrm{Aa}$ & $\mathrm{Bb}$ & & & & & & \\
\hline \multirow[t]{2}{*}{ F3 } & 30,88 & 25,00 & 2,34 & 3,73 & 3,25 & $2,86 \mathrm{Ba}$ & 0,31 & 4,49 \\
\hline & $\mathrm{Aa}$ & $\mathrm{Bb}$ & & & $\mathrm{Aab}$ & & & \\
\hline \multirow[t]{2}{*}{$\mathrm{F} 4$} & 30,30 & 25,9 & 5,95 & 9,42 & 3,29 & $3,0 \mathrm{Aa}$ & 0,35 & 4,93 \\
\hline & $\mathrm{Aa}$ & $\mathrm{Aab}$ & & & $\mathrm{Aab}$ & & & \\
\hline \multirow[t]{2}{*}{ FD } & 34,35 & 27,90 & 2,23 & 3,19 & $3,41 \mathrm{Aa}$ & $3,12 \mathrm{Aa}$ & 0,47 & 6,34 \\
\hline & $\mathrm{Aa}$ & $\mathrm{Ba}$ & & & & & & \\
\hline D.M.S. & 4,28 & 2,06 & & & 0,37 & 0,39 & & \\
\hline \multirow{2}{*}{ C.V. } & 6,23 & 3,58 & & & 4,99 & 5,80 & & \\
\hline & \multicolumn{4}{|c|}{ Rel H:D } & \multicolumn{4}{|c|}{$\mathrm{AF}\left(\mathrm{cm}^{2}\right)$} \\
\hline F1 & $8,70 \mathrm{Ab}$ & $8,70 \mathrm{Aa}$ & 0,69 & 3,52 & $117,32 \mathrm{Aa}$ & $88,22 \mathrm{Aa}$ & 58,92 & 25,48 \\
\hline \multirow[t]{2}{*}{$\mathrm{F} 2$} & 9,16 & $8,54 \mathrm{Aa}$ & 0,95 & 4,77 & $165,69 \mathrm{Aa}$ & $108,05 \mathrm{Aa}$ & 75,19 & 24,41 \\
\hline & $\mathrm{Aab}$ & & & & & & & \\
\hline \multirow[t]{2}{*}{ F3 } & 9,55 & $8,84 \mathrm{Aa}$ & 0,77 & 3,70 & $174,30 \mathrm{Aa}$ & $94,76 \mathrm{Ba}$ & 15,46 & 5,10 \\
\hline & $\mathrm{Aab}$ & & & & & & & \\
\hline \multirow[t]{2}{*}{$\mathrm{F} 4$} & 9,23 & $8,67 \mathrm{Aa}$ & 1,64 & 8,12 & $171,20 \mathrm{Aa}$ & $93,97 \mathrm{Ba}$ & 63,47 & 21,27 \\
\hline & $\mathrm{Aab}$ & & & & & & & \\
\hline \multirow[t]{2}{*}{ FD } & 10,13 & $8,96 \mathrm{Ba}$ & 0,92 & 4,28 & $174,85 \mathrm{Aa}$ & $93,50 \mathrm{Ba}$ & 61,24 & 20,28 \\
\hline & $\mathrm{Aa}$ & & & & & & & \\
\hline D.M.S. & 1,27 & 0,78 & & & 67,16 & 44,17 & & \\
\hline \multirow[t]{2}{*}{ C.V. } & 6,06 & 3,98 & & & 18,55 & 20,48 & & \\
\hline & \multicolumn{4}{|c|}{$\operatorname{NPF}\left(n^{0}\right)$} & \multicolumn{4}{|c|}{$\mathrm{NR}\left(\mathrm{n}^{\mathrm{o}}\right)$} \\
\hline$\overline{F 1}$ & $6,73 \mathrm{Aa}$ & $5,78 \mathrm{Aa}$ & 3,85 & 27,36 & $0,65 \mathrm{Aa}$ & $0,35 \mathrm{Aa}$ & 3,85 & 157,5 \\
\hline $\mathrm{F} 2$ & $7,85 \mathrm{Aa}$ & $4,90 \mathrm{Bb}$ & 2,74 & 19,14 & $1,35 \mathrm{Aa}$ & $0,0 \mathrm{Ba}$ & 1,23 & 80,92 \\
\hline \multirow[t]{2}{*}{ F3 } & $7,15 \mathrm{Aa}$ & 4,98 & 1,09 & 7,99 & $0,90 \mathrm{Aa}$ & $0,0 \mathrm{Ba}$ & 0,80 & 79,09 \\
\hline & & $\mathrm{Bab}$ & & & & & & \\
\hline \multirow[t]{2}{*}{$\mathrm{F} 4$} & $6,73 \mathrm{Aa}$ & 5,12 & 2,40 & 18,0 & $0,85 \mathrm{Aa}$ & $0,0 \mathrm{Aa}$ & 0,88 & 91,63 \\
\hline & & $\mathrm{Aab}$ & & & & & & \\
\hline FD & $8,35 \mathrm{Aa}$ & 5,45 & 0,79 & 5,09 & $1,80 \mathrm{Aa}$ & $0,05 \mathrm{Ba}$ & 1,63 & 78,33 \\
\hline & & $\mathrm{Bab}$ & & & & & & \\
\hline D.M.S. & 2,91 & 0,83 & & & 1,88 & 0,37 & & \\
\hline C.V. & 17,54 & 7,02 & & & 75,21 & 202,84 & & \\
\hline
\end{tabular}

D.M.S.: diferença mínima estatística do Teste de Tukey $(\alpha=5 \%)$; C.V.: coeficiente de variação. Médias seguidas de letras minúsculas iguais na mesma coluna e maiúsculas iguais na mesma linha, não diferem pelo Teste de Tukey, ao nível de 5\% de significância. 
Estes resultados indicam que as práticas de campo relativas à implantação e ao manejo (Barros et al., 1990; Stape et al., 2001; Gava, 2002; Neves, 2004) seguidas nesta pesquisa, se sobressaíssem em relação à qualidade das mudas, no que tange à aclimatação em viveiro através do manejo da água, possibilitando que o solo arenoso, menos fértil e com menor índice pluviométrico total (Figura 2), garantisse mesmo ritmo de crescimento à plantação nos períodos avaliados, aos seis e aos 13 meses de idade. Além disso, os índices pluviométricos anuais acumulados em 2006 de cada um dos locais, de $2.269,5 \mathrm{~mm}$ para o solo argiloso e de $2.107,3$ para o arenoso garantiram que não faltasse água tanto em um local como no outro.

Tabela 6. Resultados médios das alturas de Eucalyptus grandis vs. Eucalyptus urophylla, aos 6 meses de idade, em função do substrato e do manejo hídrico.

\begin{tabular}{|c|c|c|c|c|c|c|c|c|}
\hline \multirow{4}{*}{$\begin{array}{l}\text { Manejo } \\
\text { hídrico } \\
\text { de } \\
\text { viveiro }\end{array}$} & \multicolumn{8}{|c|}{ Altura (m) } \\
\hline & \multirow{2}{*}{\multicolumn{4}{|c|}{$\frac{\text { Solo de textura arenosa }}{\text { Substrato }}$}} & \multicolumn{4}{|c|}{ Solo de textura argilosa } \\
\hline & & & & & & & rato & \\
\hline & $\mathrm{CACV}$ & PLX & D.M.S. & C.V. & $\mathrm{CACV}$ & PLX & D.M.S. & C.V. \\
\hline $\mathrm{F} 1$ & $0,86 \mathrm{Aa}$ & $0,94 \mathrm{Aa}$ & 0,29 & 14,33 & $0,76 \mathrm{Aa}$ & $\begin{array}{c}1,14 \\
\mathrm{Aa}\end{array}$ & 0,55 & 25,88 \\
\hline $\mathrm{F} 2$ & $1,02 \mathrm{Aa}$ & $0,93 \mathrm{Aa}$ & 0,26 & 11,84 & $0,82 \mathrm{Aa}$ & $\begin{array}{c}1,13 \\
\mathrm{Aa}\end{array}$ & 0,90 & 41,02 \\
\hline F3 & $0,96 \mathrm{Aa}$ & 0,88 Aa & 0,21 & 10,08 & $0,92 \mathrm{Aa}$ & $\begin{array}{c}0,88 \\
\mathrm{Aa}\end{array}$ & 0,10 & 5,04 \\
\hline F4 & $1,10 \mathrm{Aa}$ & $0,99 \mathrm{Aa}$ & 0,30 & 12,55 & $1,00 \mathrm{Aa}$ & $\begin{array}{c}0,83 \\
\mathrm{Aa}\end{array}$ & 0,32 & 15,57 \\
\hline FD & $1,15 \mathrm{Aa}$ & $1,03 \mathrm{Aa}$ & 0,28 & 11,23 & $0,94 \mathrm{Aa}$ & $\begin{array}{c}0,93 \\
\mathrm{Aa}\end{array}$ & 0,26 & 12,56 \\
\hline D.M.S. & 0,29 & 0,42 & & & 0,34 & 0,62 & & \\
\hline C.V. & 12,74 & 19,69 & & & 16,84 & 27,94 & & \\
\hline
\end{tabular}

D.M.S.: diferença mínima estatística do Teste de Tukey $(\alpha=5 \%)$ C.V.: coeficiente de variação. Médias seguidas de letras minúsculas iguais na mesma coluna e maiúsculas iguais na mesma linha, para o mesmo solo, não diferem pelo Teste de Tukey, ao nível de 5\% de significância.

Tabela 7. Resultados médios das alturas de Eucalyptus grandis vs. Eucalyptus urophylla, aos 6 meses de idade, em função do manejo hídrico para os tipos de solos.

\begin{tabular}{ccccc}
\hline \multirow{2}{*}{$\begin{array}{c}\text { Manejo hídrico } \\
\text { de viveiro }\end{array}$} & \multicolumn{4}{c}{ Altura $(\mathrm{m})$} \\
\cline { 2 - 5 } & Textura arenosa & Textura argilosa & D.M.S. & C.V. \\
\hline F1 & 0,90 A & $0,95 \mathrm{~A}$ & 0,23 & 22,08 \\
F2 & 0,98 A & $0,98 \mathrm{~A}$ & 0,32 & 29,17 \\
F3 & 0,92 A & $0,90 \mathrm{~A}$ & 0,08 & 8,32 \\
F4 & 1,04 A & $0,92 \mathrm{~A}$ & 0,20 & 18,64 \\
FD & 1,09 A & $0,94 \mathrm{~A}$ & 0,13 & 11,43 \\
\hline
\end{tabular}

D.M.S.: Diferença mínima estatística do Teste de Tukey $(\alpha=5 \%)$ C.V.: coeficiente de variação. Médias seguidas de letras maiúsculas iguais na mesma linha, não diferem pelo Teste de Tukey, ao nível de 5\% de significância. 
Com relação ao desenvolvimento em diâmetro das árvores aos 13 meses de idade (Tabelas 8 e 9), verifica-se o mesmo comportamento observado para o desenvolvimento em altura, ou seja, não houve influência dos substratos usados na produção e da aclimatação realizada durante a fase de rustificação, bem como do regime de chuvas nos dois locais de plantio. Assim também verificaram Rezende et al. (1984, apud Stape et al., 2001), aos 60 meses de idade em um plantio de eucalipto realizado com mudas rustificadas e não rustificadas em viveiro, e diferentemente do que observaram Souza et al. (2006) em três regiões da Bacia do Rio Doce, $\mathrm{MG}$.

Tabela 8. Resultados médios das alturas e dos diâmetros de Eucalyptus grandis vs. Eucalyptus urophylla aos treze meses de idade, em função do substrato, do manejo hídrico e do tipo de solo.

\begin{tabular}{|c|c|c|c|c|c|c|c|c|}
\hline \multirow{4}{*}{$\begin{array}{l}\text { Manejo } \\
\text { hídrico } \\
\text { de } \\
\text { viveiro }\end{array}$} & \multicolumn{8}{|c|}{ Altura (m) } \\
\hline & \multirow{2}{*}{\multicolumn{4}{|c|}{$\begin{array}{c}\text { Solo de textura arenosa } \\
\text { Substrato }\end{array}$}} & \multicolumn{4}{|c|}{ Solo de textura argilosa } \\
\hline & & & & & \multicolumn{4}{|c|}{ Substrato } \\
\hline & $\mathrm{CACV}$ & PLX & D.M.S. & $\mathrm{CV}$ & $\mathrm{CACV}$ & PLX & D.M.S. & $\mathrm{CV}$ \\
\hline $\mathrm{F} 1$ & $4,25 \mathrm{Aab}$ & 4,39 $\mathrm{Aa}$ & 0,77 & 7,88 & $3,76 \mathrm{Ba}$ & $\begin{array}{c}4,97 \\
\mathrm{Aa}\end{array}$ & 0,89 & 9,04 \\
\hline $\mathrm{F} 2$ & $4,14 \mathrm{Aab}$ & $4,44 \mathrm{Aa}$ & 0,42 & 4,37 & $4,02 \mathrm{Aa}$ & $\begin{array}{c}4,68 \\
\text { Aa }\end{array}$ & 2,00 & 20,43 \\
\hline F3 & 4,45 Aab & $4,38 \mathrm{Aa}$ & 0,67 & 6,70 & $4,35 \mathrm{Aa}$ & $\begin{array}{c}4,19 \\
\text { Aa }\end{array}$ & 0,28 & 2,95 \\
\hline F4 & $4,32 \mathrm{Ab}$ & 4,49 Aa & 0,51 & 5,14 & 4,52 $\mathrm{Aa}$ & $\begin{array}{c}4,09 \\
\mathrm{Aa}\end{array}$ & 0,75 & 7,77 \\
\hline FD & 4,86 $\mathrm{Aa}$ & 4,64 Aa & 0,18 & 1,65 & 4,47 Aa & $\begin{array}{c}4,33 \\
\mathrm{Aa} \\
\end{array}$ & 1,01 & 10,18 \\
\hline D.M.S. & 0,52 & 0,88 & & & 1,16 & 1,45 & & \\
\hline \multirow[t]{2}{*}{ C.V. } & 5,20 & 8,79 & & & 12,15 & 14,46 & & \\
\hline & \multicolumn{8}{|c|}{ Diâmetro $(\mathrm{cm})$} \\
\hline $\mathrm{F} 1$ & $3,41 \mathrm{Ab}$ & $3,56 \mathrm{Aa}$ & 0,95 & 12,16 & $3,04 \mathrm{Aa}$ & $\begin{array}{c}4,44 \\
\mathrm{Aa}\end{array}$ & 0,89 & 9,04 \\
\hline $\mathrm{F} 2$ & $3,31 \mathrm{Bb}$ & 3,75 Aa & 0,36 & 4,49 & $3,30 \mathrm{Aa}$ & $\begin{array}{c}4,21 \\
\mathrm{Aa}\end{array}$ & 2,00 & 20,43 \\
\hline F3 & $3,70 \mathrm{Aab}$ & $3,49 \mathrm{Aa}$ & 0,62 & 7,70 & $3,55 \mathrm{Aa}$ & $\begin{array}{c}3,56 \\
\mathrm{Aa}\end{array}$ & 0,28 & 2,95 \\
\hline F4 & 3,66 Aab & 3,87 Aa & 0,55 & 6,50 & 3,86 Aa & $\begin{array}{c}3,42 \\
\mathrm{Aa}\end{array}$ & 0,75 & 7,77 \\
\hline FD & $4,16 \mathrm{Aa}$ & $4,04 \mathrm{Aa}$ & 0,39 & 4,21 & 4,01 Aa & $\begin{array}{c}3,73 \\
\mathrm{Aa}\end{array}$ & 1,01 & 10,18 \\
\hline D.M.S. & 0,58 & 1,02 & & & 1,25 & 1,93 & & \\
\hline C.V. & 7,08 & 12,08 & & & 15,65 & 22,16 & & \\
\hline
\end{tabular}

D.M.S.: diferença mínima estatística do Teste de Tukey $(\alpha=5 \%)$; C.V.: coeficiente de variação. Médias seguidas de letras minúsculas iguais na mesma coluna e maiúsculas iguais na mesma linha, para o mesmo solo, não diferem pelo Teste de Tukey, ao nível de $5 \%$ de significância. 
Embora não tenha sido realizada a avaliação do desenvolvimento no primeiro mês de estabelecimento da floresta, poderia se esperar que os efeitos do manejo de viveiro, no presente estudo, referentes ao substrato, à aclimatação através do manejo hídrico e aos índices pluviométricos dos dois locais de plantio no período avaliado, pudessem exercer influência no crescimento, conforme relata Neves (2004). Porém, na pesquisa de Rezende et al. (1984, apud Stape et al., 2001), essa diferença não aconteceu desde a idade zero do estabelecimento da floresta. Nessa pesquisa, os índices mensais de chuva de dezembro de 2006 e de janeiro de 2007 foram significativamente elevados quando comparados à média histórica do estado de SP. Mesmo tendo sido maiores na área argilosa, de $606,5 \mathrm{~mm}$ e $738 \mathrm{~mm}$ mensais, respectivamente, quando comparados aos da área arenosa, de 343,8 e de $601,5 \mathrm{~mm}$ mensais, respectivamente (Figura 2), possivelmente, também não resultarão em ritmos distintos de crescimento da plantação, pois novamente o suprimento de água se encontra acima do requerido. É esperado que quando os índices pluviométricos forem abaixo do requerido para um dos dois locais, os ritmos de crescimento sejam distintos, mesmo com aporte nutricional adequado.

Tabela 9. Resultados médios das alturas e dos diâmetros das árvores de Eucalyptus grandis vs. Eucalyptus urophylla, aos 13 meses de idade, em função do manejo hídrico para os tipos de solos.

\begin{tabular}{|c|c|c|c|c|}
\hline \multirow{3}{*}{$\begin{array}{l}\text { Manejo hídrico de } \\
\text { viveiro }\end{array}$} & \multicolumn{4}{|c|}{ Altura (m) } \\
\hline & \multicolumn{4}{|c|}{ Tipo de solo } \\
\hline & textura arenosa & $\begin{array}{c}\text { textura } \\
\text { argilosa }\end{array}$ & D.M.S. & C.V. \\
\hline F1 & $4,37 \mathrm{Aa}$ & $4,32 \mathrm{Aa}$ & 0,52 & 10,72 \\
\hline $\mathrm{F} 2$ & 4,29 Aa & $4,35 \mathrm{Aa}$ & 0,65 & 13,45 \\
\hline F3 & $4,41 \mathrm{Aa}$ & $4,28 \mathrm{Aa}$ & 0,49 & 10,17 \\
\hline $\mathrm{F} 4$ & $4,41 \mathrm{Aa}$ & $4,30 \mathrm{Aa}$ & 0,49 & 10,16 \\
\hline \multirow[t]{2}{*}{ FD } & $4,75 \mathrm{Aa}$ & $4,40 \mathrm{Ba}$ & 0,33 & 6,53 \\
\hline & \multicolumn{4}{|c|}{ Diâmetro (cm) } \\
\hline F1 & $3,74 \mathrm{Aa}$ & $3,48 \mathrm{Aa}$ & 0,68 & 16,91 \\
\hline $\mathrm{F} 2$ & $3,53 \mathrm{Aa}$ & $3,75 \mathrm{Aa}$ & 0,78 & 19,13 \\
\hline F3 & $3,60 \mathrm{Aa}$ & $3,56 \mathrm{Aa}$ & 0,58 & 14,48 \\
\hline $\mathrm{F} 4$ & $3,77 \mathrm{Aa}$ & $3,64 \mathrm{Aa}$ & 0,61 & 14,87 \\
\hline FD & $4,10 \mathrm{Aa}$ & $3,87 \mathrm{Aa}$ & 0,46 & 10,42 \\
\hline
\end{tabular}

D.M.S.: diferença mínima estatística do Teste de Tukey $(\alpha=5 \%)$; C.V.: coeficiente de variação. Médias seguidas de letras maiúsculas iguais na mesma linha não diferem pelo Teste de Tukey, ao nível de 5\% de significância.

\section{CONCLUSÃO}

A aclimatação em viveiro das mudas de Eucalyptus grandis vs. Eucalyptus urophylla produzidas nos substratos CACV e PLX, realizada pelo manejo da água durante a fase de rustificação das mudas, não exerceu influência no crescimento em altura e em diâmetro das árvores aos seis e aos 13 meses de idade, tanto no solo de textura arenosa, como no de textura argilosa, para as condições desta pesquisa. 


\section{REFERÊNCIAS}

BARROS, N. F.; NOVAIS, R. F.; NEVES, J. C. L. Fertilização e correção do solo para o plantio de eucalipto. In: BARROS, N. F.; NOVAIS, R. F. (Ed.). Relação solo-eucalipto. Viçosa: Editora Folha de Viçosa, 1990, p. 127-186.

CARNEIRO, J. G. A. Produção e controle de qualidade de mudas florestais. Curitiba: UFPR/FUPEF., 1995. $451 \mathrm{p}$.

CARVALHO, A. M. Valorização da madeira do híbrido Eucalyptus grandis x Eucalyptus urophylla através da produção conjunta de madeira serrada em pequenas dimensões, celulose e lenha. 2000. 129 p. Dissertação (Mestrado em Ciências e Tecnologia da Madeira) - Escola Superior de Agricultura “Luiz de Queiroz”, Universidade de São Paulo, Piracicaba, 2000.

COUTO, L. Cultivation and production of eucalypts in South América: with special reference to the leaf oils. In: COPPEN, J. J. W. (Ed.). Eucalyptus: the genus Eucalyptus. Taylor \& Francis: London, 2002 p. 239-250.

GAVA, J. L. Cultivo mínimo de solos com textura arenosa e média em áreas planas e suaveonduladas. In: GONÇALVES, J. L. de M.; STAPE, J. L. (Ed.). Conservação e cultivo de solos para plantações florestais. IPEF: Piracicaba, 2002 p. 223-243.

GONÇALVES, J. L. de M.; RAIJ, B.; GONÇALVES, J. C. Florestais. Boletim IAC, Campinas, n. 100, p. 245-259, 1996.

LOPES, J. L. W.; GUERRINI, I. A.; SAAD, J. C. C.; SILVA, M. R. da. Efeitos da irrigação na sobrevivência, transpiração e no teor relativo de água na folha em mudas de Eucalyptus grandis em diferentes substratos. Scientia Forestalis, Piracicaba, n. 68, p. 97-106, 2005.

NEVES, J. C. L. Doses e modos de localização dos nutrientes em recipientes contendo volumes variáveis de substrato. In: ENCONTRO NACIONAL SOBRE SUBSTRATO PARA PLANTAS, 4., 2004, Viçosa, Anais... Viçosa: Universidade Federal de Viçosa, 2004. Viçosa, 2004.

PEREIRA, M. R. R.; et. al. Comportamento fisiológico e morfológico de clones de Eucalyptus urograndis submetidos à diferentes níveis de água no solo. Irriga, Botucatu, v. 11, n. 4, p. 518-531, 2006.

SASSE, J., et.al. Comparative responses of cuttings and seedlings of Eucalyptus grandis of Eucalyptus globulus to water stress. Tree Physiology, Victoria, v. 16, p. 287-294, 1996.

SILVA, J. C.; MATOS, J. L. M. A madeira de eucalipto na indústria moveleira. Revista da Madeira, Curitiba, n. 70, p. 36-40, 2003.

SILVA, M. R. da.; KLAR, A. E.; PASSOS, J. R. Efeitos do manejo hídrico e da aplicação de potássio nas características morfofisiológicas de mudas de Eucalyptus grandis W. (Hill ex. Maiden). Irriga, Botucatu, v. 9, n. 1, 2004, p. 31-40. 
SOUZA, M. J. H.; et.al. Disponibilidade hídrica do solo e produtividade do eucalipto em três regiões da Bacia do Rio Doce. Revista Árvore, Viçosa, v. 30, n. 3, p. 399-410, 2006.

STAPE, J. L.; GONÇALVES, J. L. de M.; GONÇALVES, A. N. Relationships between nursery practices and field performance for Eucalyptus plantations in Brazil. New Forests, Netherlands, n. 22, p. 19-41, 2001. 